\title{
Rearrangement of Indene Skeletons under Mild Conditions
}

Yoichiro Kuninobu,* Hirokazu Ueda, Atsushi Kawata, and Kazuhiko Takai*

Division of Chemistry and Biochemistry, Graduate School of Natural Science and Technology, Okayama University, Tsushima, Okayama 700-8530, Japan

E-mail: kuninobu@cc.okayama-u.ac.jp; ktakai@cc.okayama-u.ac.jp

\section{Table of Contents}

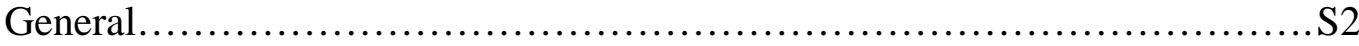

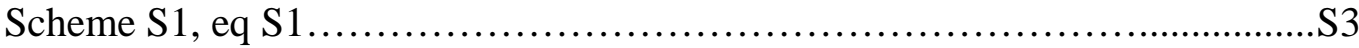

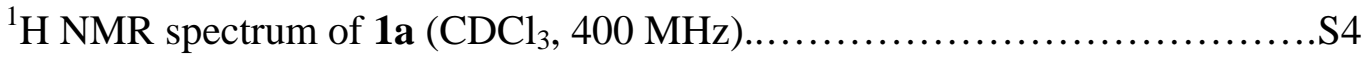

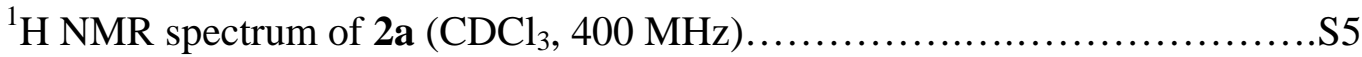

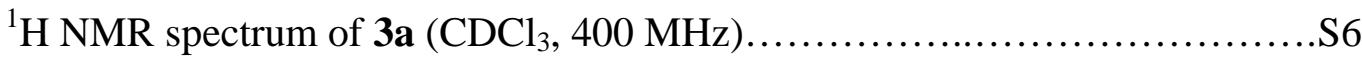

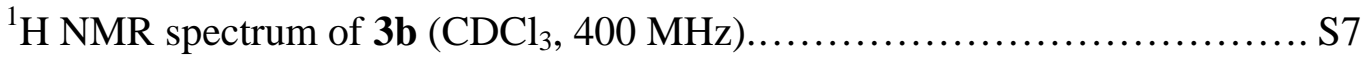

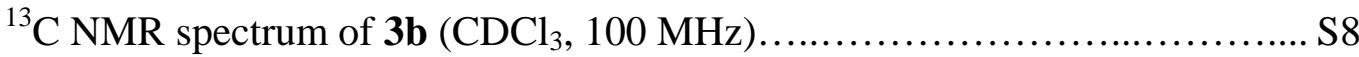

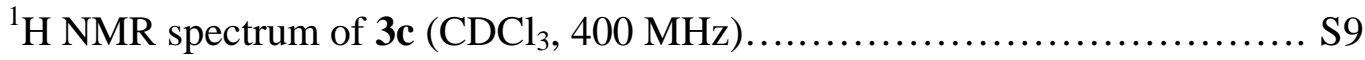

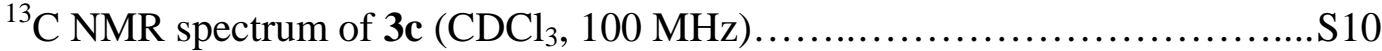

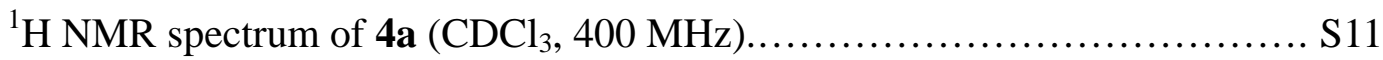

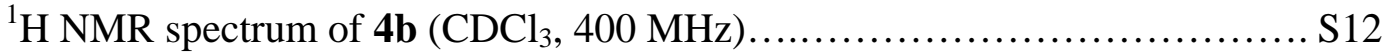

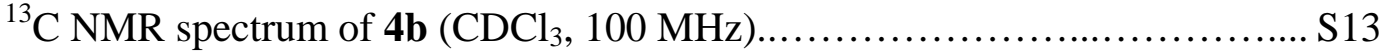



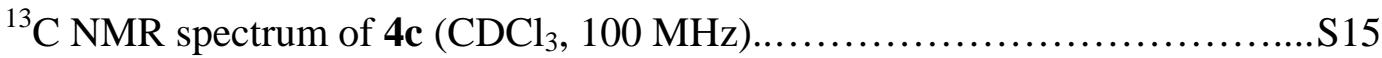

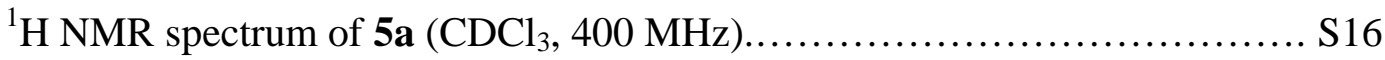

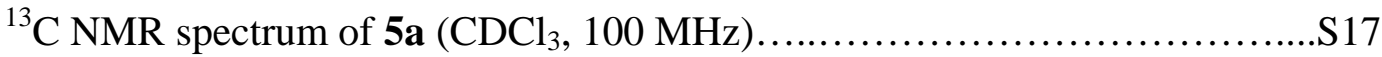

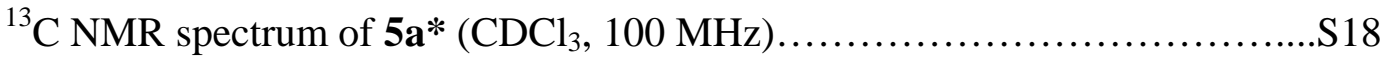

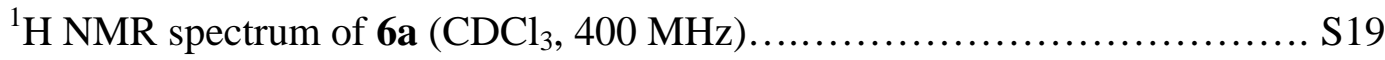

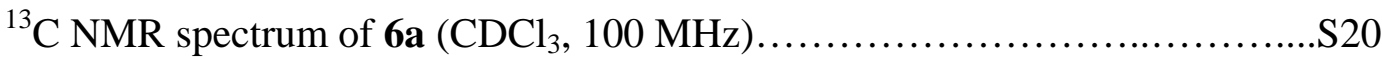

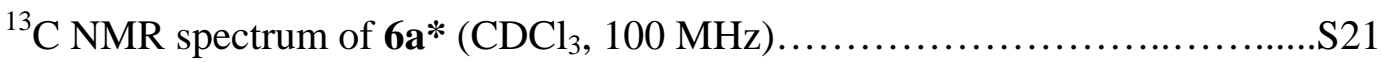


General. All reactions were carried out in a dry solvent under an argon atmosphere.

Diethyl ether, acetonitrile, tetrahydrofuran (THF), toluene and $N, N$-dimethylformamide (DMF) were purchased from Wako Pure Chemical Industries or Kanto Kagaku Co., and were dried, distilled and degassed before use. Aminoindene derivatives were prepared according to the literature method. ${ }^{10}$ Iodomethane $-{ }^{13} \mathrm{C}$ was purchased from ISOTEC Co., and used without purification.

${ }^{1} \mathrm{H}$ (400 MHz) and ${ }^{13} \mathrm{C}$ (100 MHz) NMR spectra were recorded using a JEOL JNM-LA400 spectrometer. Proton chemical shifts are reported relative to $\mathrm{Me}_{4} \mathrm{Si}$ $\left(\mathrm{CDCl}_{3}\right)$ at $\delta 0.00 \mathrm{ppm}$ or residual solvent peak $\left(\mathrm{CDCl}_{3}\right.$ at $\left.\delta 7.26 \mathrm{ppm}\right)$. Carbon chemical shifts are reported relative to $\mathrm{CDCl}_{3}$ at $\delta 77.00 \mathrm{ppm}$. IR spectra were recorded on a Nicolet Protégé 460. 
Scheme S1. Preparation of ${ }^{13} \mathrm{C}$-labeled 1-Phenyl-1-octyne<smiles>CCOC(C)[C@H](C)[C@H](C)OCC</smiles>

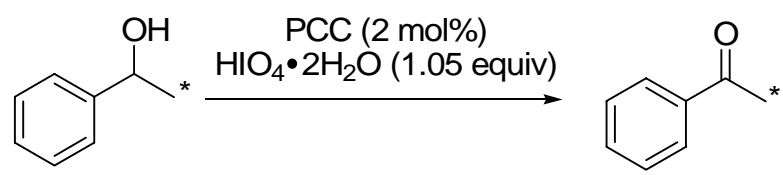

1, LDA (1.05 equiv)

2, $\operatorname{CIOP}(\mathrm{OEt})_{2}$ (1.1 equiv)

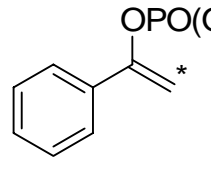

1, ${ }^{n}$ BuLi (1.1 equiv) $\mathrm{THF},-78^{\circ} \mathrm{C}, 3 \mathrm{~h}$

2, ${ }^{n} \mathrm{C}_{6} \mathrm{H}_{13} \mathrm{l}$ (1.1 equiv) THF, $60^{\circ} \mathrm{C}, 12 \mathrm{~h}$<smiles>C(#Cc1ccccc1)c1ccccc1</smiles>

2, aq. $\mathrm{HCl}$<smiles>C#Cc1ccccc1</smiles><smiles>CC(C)(C)N=Cc1ccccc1</smiles><smiles>C(C#Cc1ccccc1)#Cc1ccccc1</smiles>

$\left[\operatorname{ReBr}(\mathrm{CO})_{3}(\text { thf })\right]_{2}$ $\underset{\text { toluene, } 135^{\circ} \mathrm{C}, 1 \mathrm{~h}}{\longrightarrow}$<smiles>CC(C)(C)CCCCCCCCCCCCC(=O)NC1=C(c2ccccc2)c2ccccc21</smiles>

$59 \%$<smiles>CC(C)(C)NC1=C(c2ccccc2)[C+](c2ccccc2)c2ccccc21</smiles>

$39 \%$ 

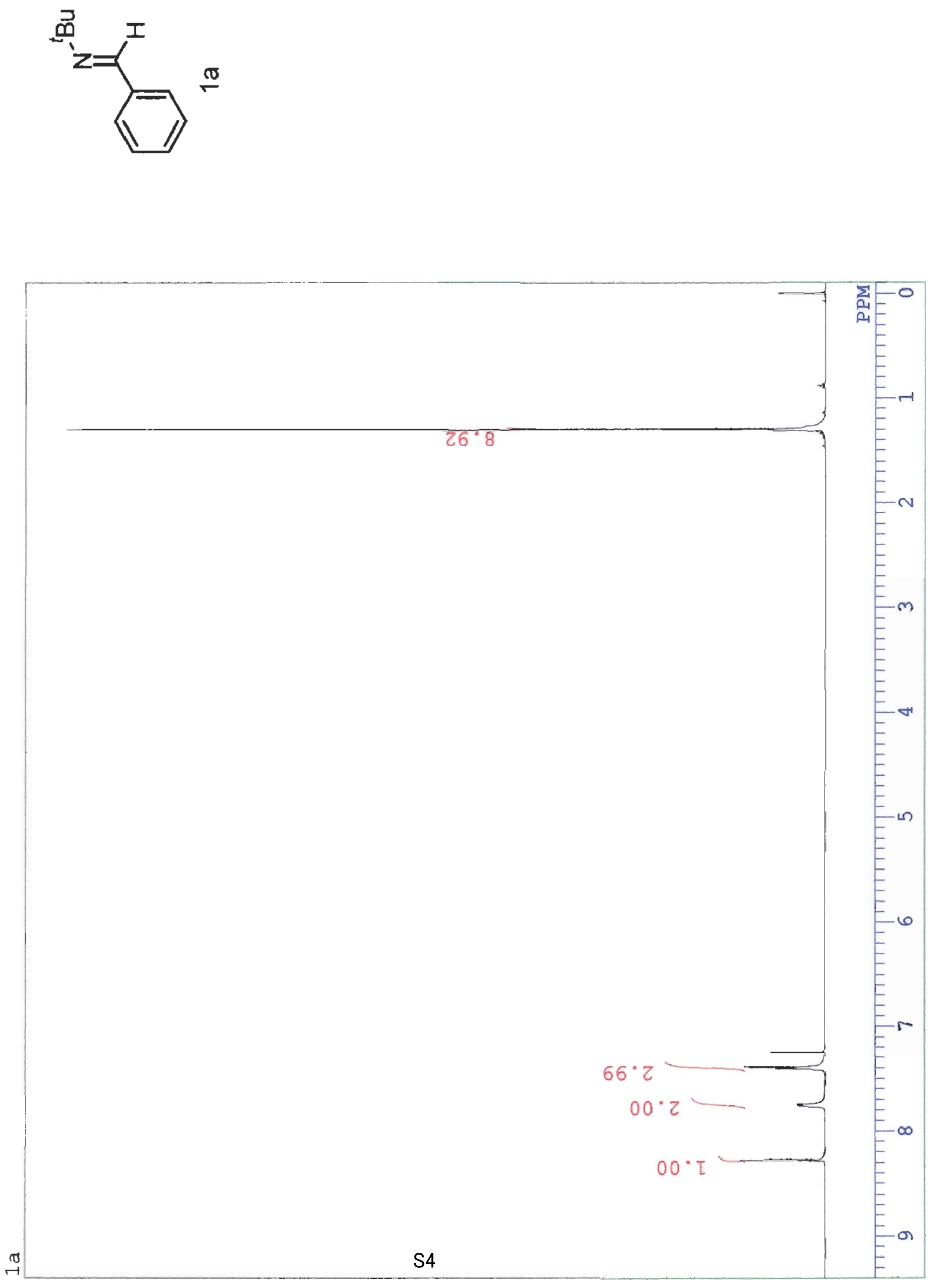


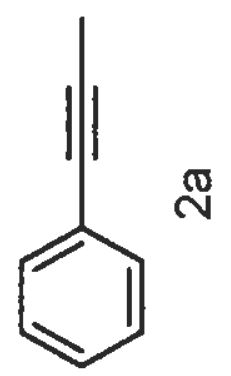

989ع $0^{\circ} 2$

$98100^{\circ} \mathrm{C}$

$98070^{\circ} z$

$29602^{\circ} L$

LE992 $L$

$\angle 8092$

LES92 $2^{\circ} L$

[โ692 $2^{\circ} \mathrm{L}$

I9\&L乙

II LL $\angle L$

$607 L \varepsilon$

6ฐ $8 L \varepsilon$

$60 \varepsilon 8 \varepsilon$

$6 S L 8 \varepsilon$

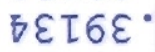

ฤ $९$ $6 \varepsilon^{\circ}$

Б8โОБ $L$ 


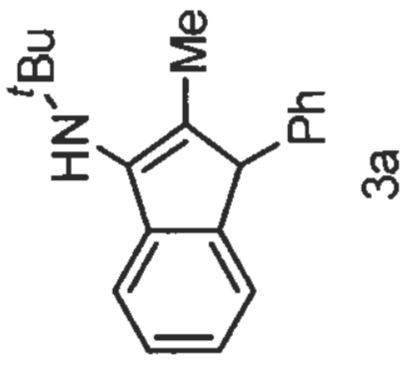

$\frac{\angle S Z D 6^{\circ} 0}{\angle 0 D \square 6^{\circ} 0}$

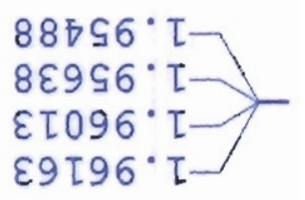

$\angle \varepsilon 8$ โ9

乙โZ29

68๐Eโ $L \longrightarrow$

DILET:L-

DIGSI:L-

万99SI $L$

$\varepsilon\left[6 L I^{\circ} L\right]$

8ह[8 [ $L]$

$8828\left[^{\circ} L\right.$

$88002^{\circ} \mathrm{L}-$

$\angle 882^{\circ} \mathrm{L}-$

$29922^{\circ} L-$

$\angle 8 L^{\circ} Z^{\circ}$

$\angle \varepsilon 622^{\circ} L$

LESE $Z^{\circ} L$

$\left\llcorner\varepsilon 8 \varepsilon Z^{\circ} L-F\right.$

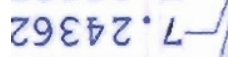

LELDZ $L$

$9 \varepsilon[0 \varepsilon \cdot L-$

$098 \tau \varepsilon \cdot L-$

09โZE $L$ -

$096 \varepsilon \varepsilon^{\circ} L-$

$80 \angle 90^{\circ} L-$ 

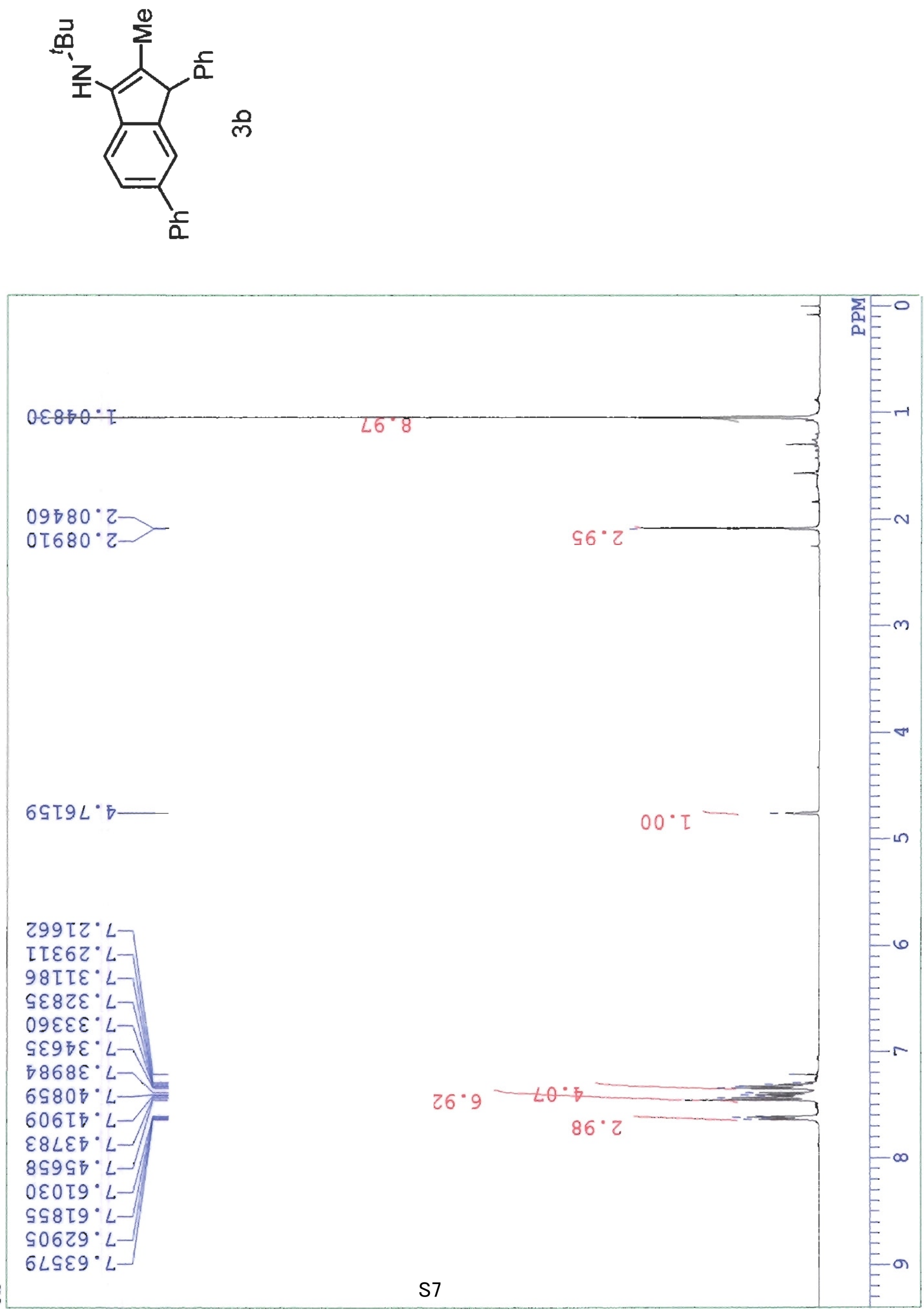

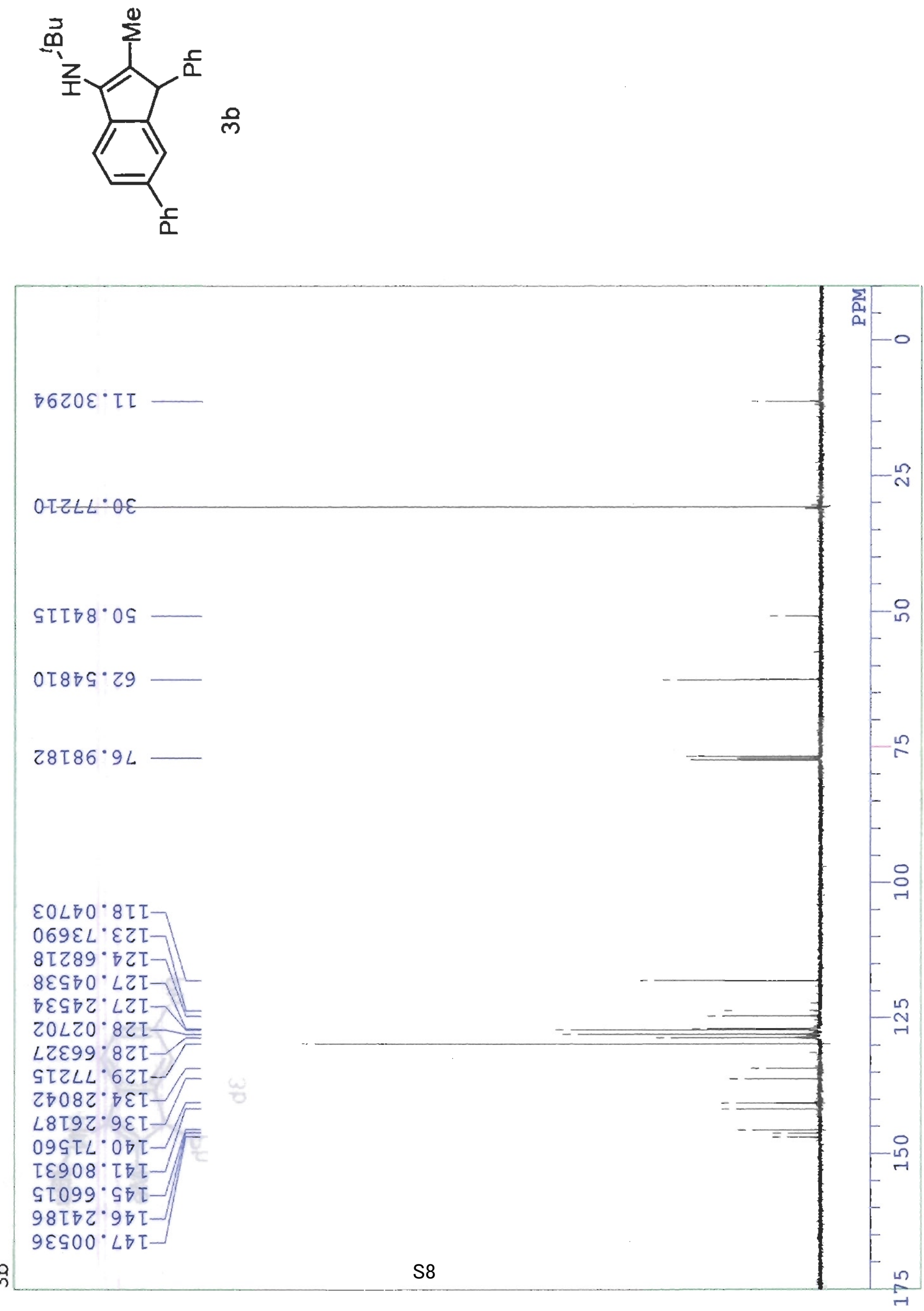

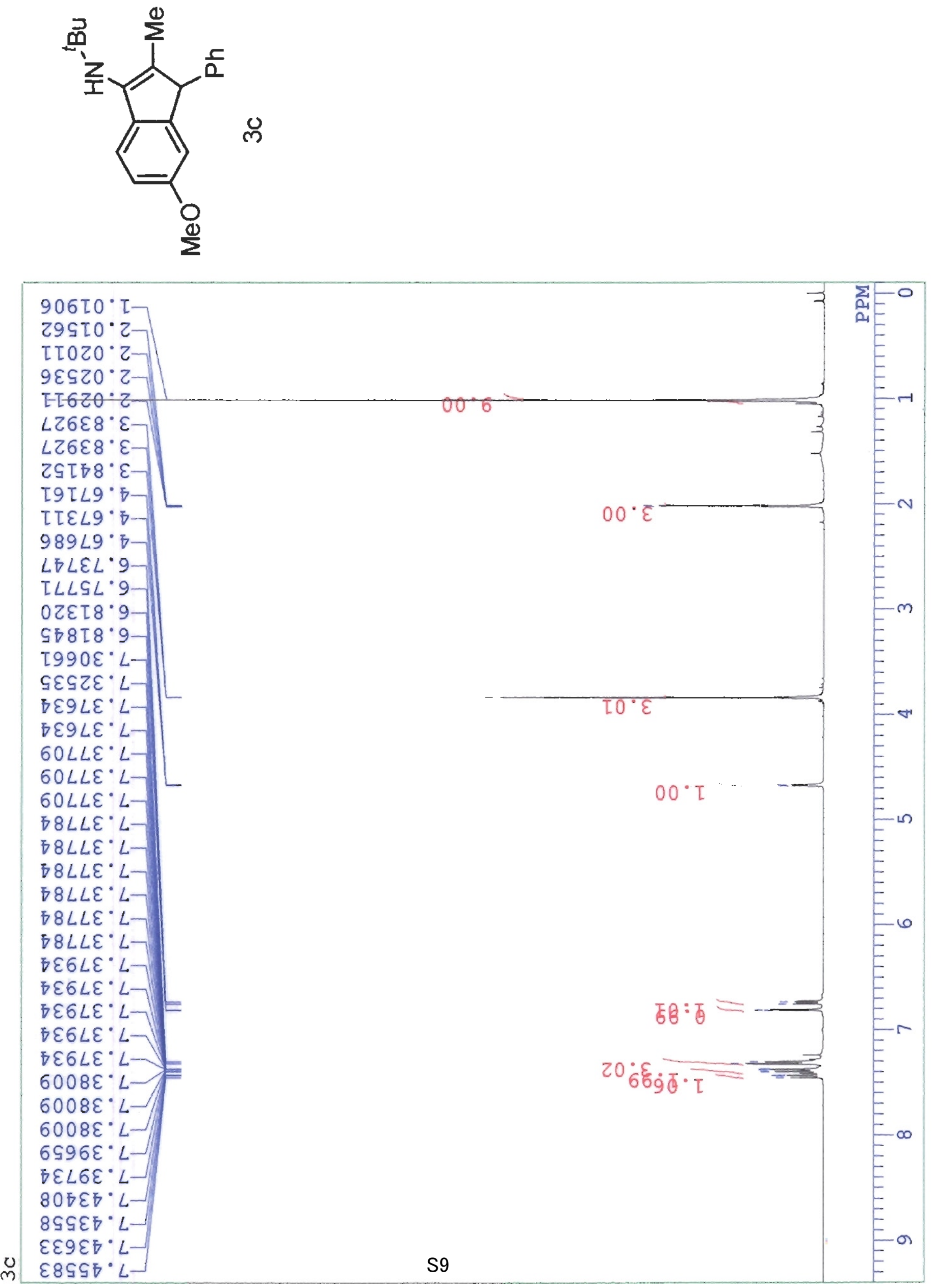

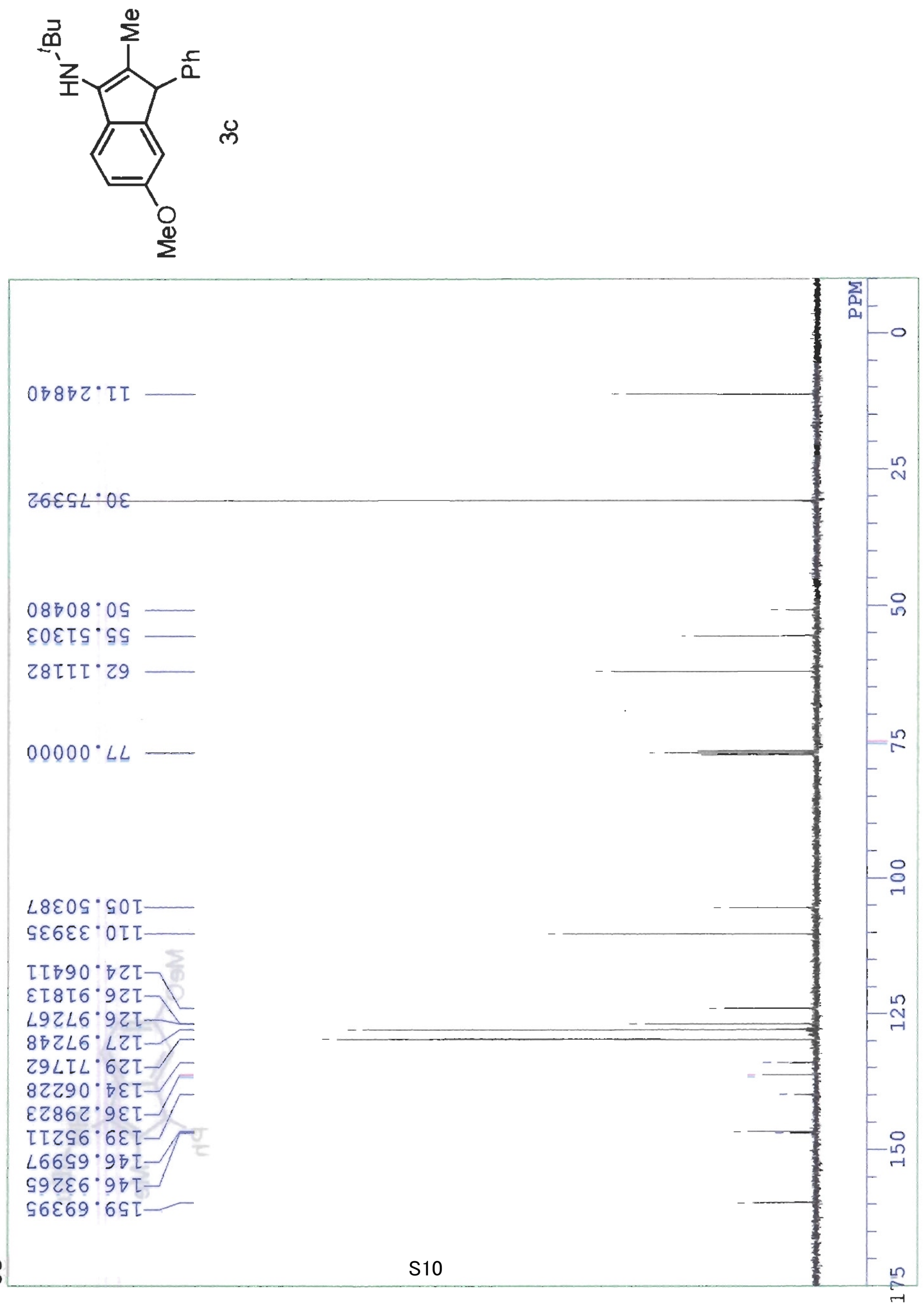

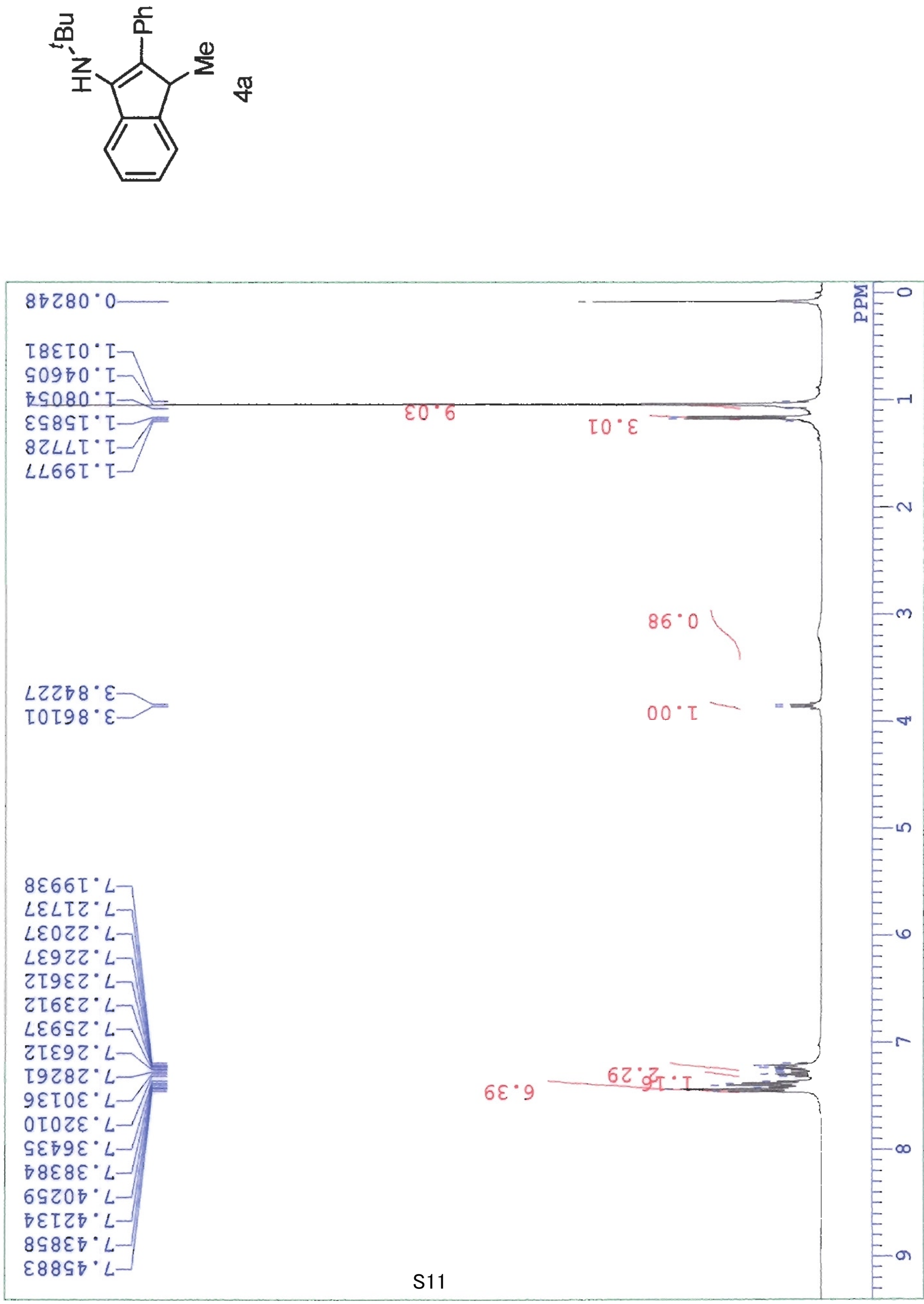

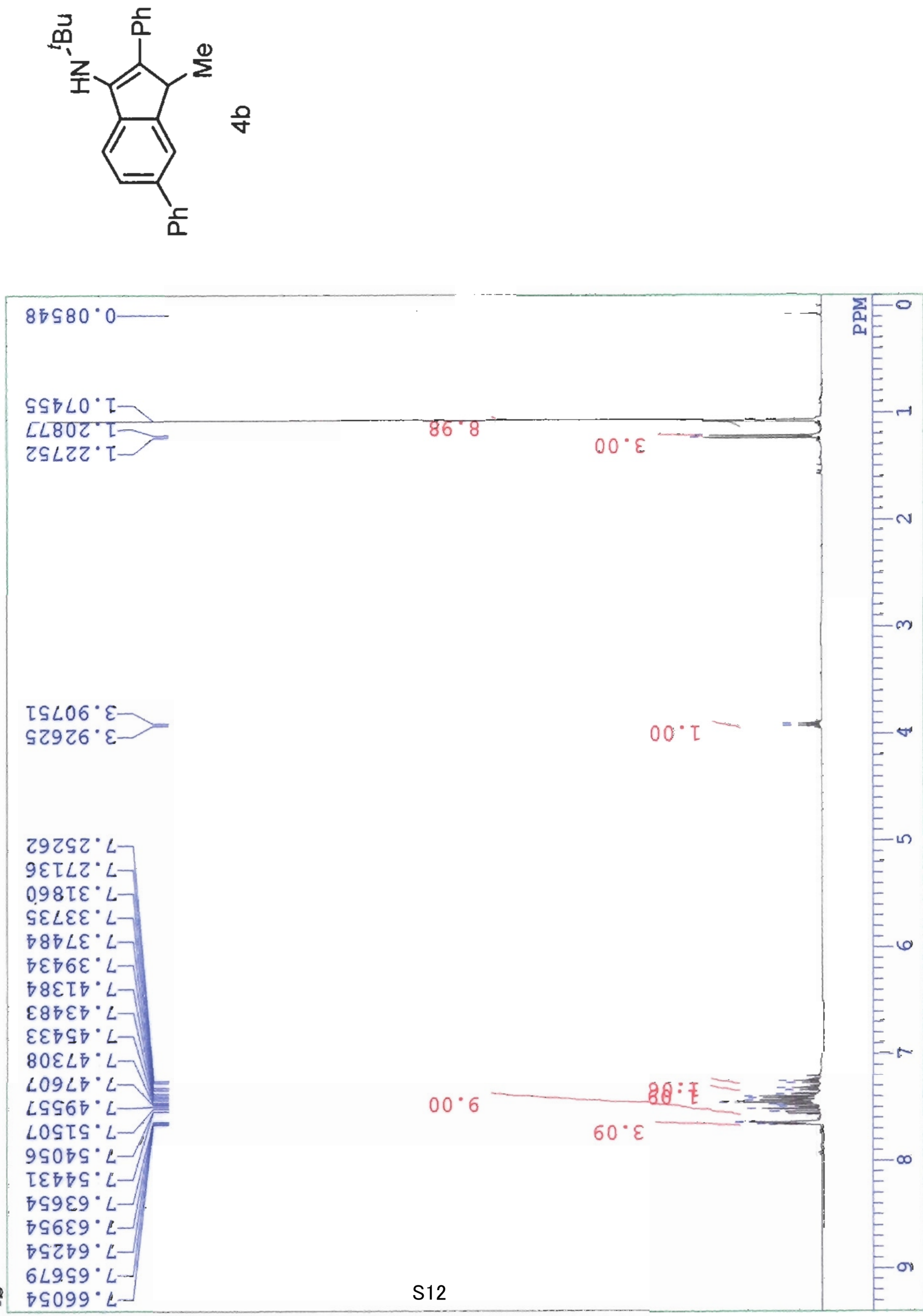

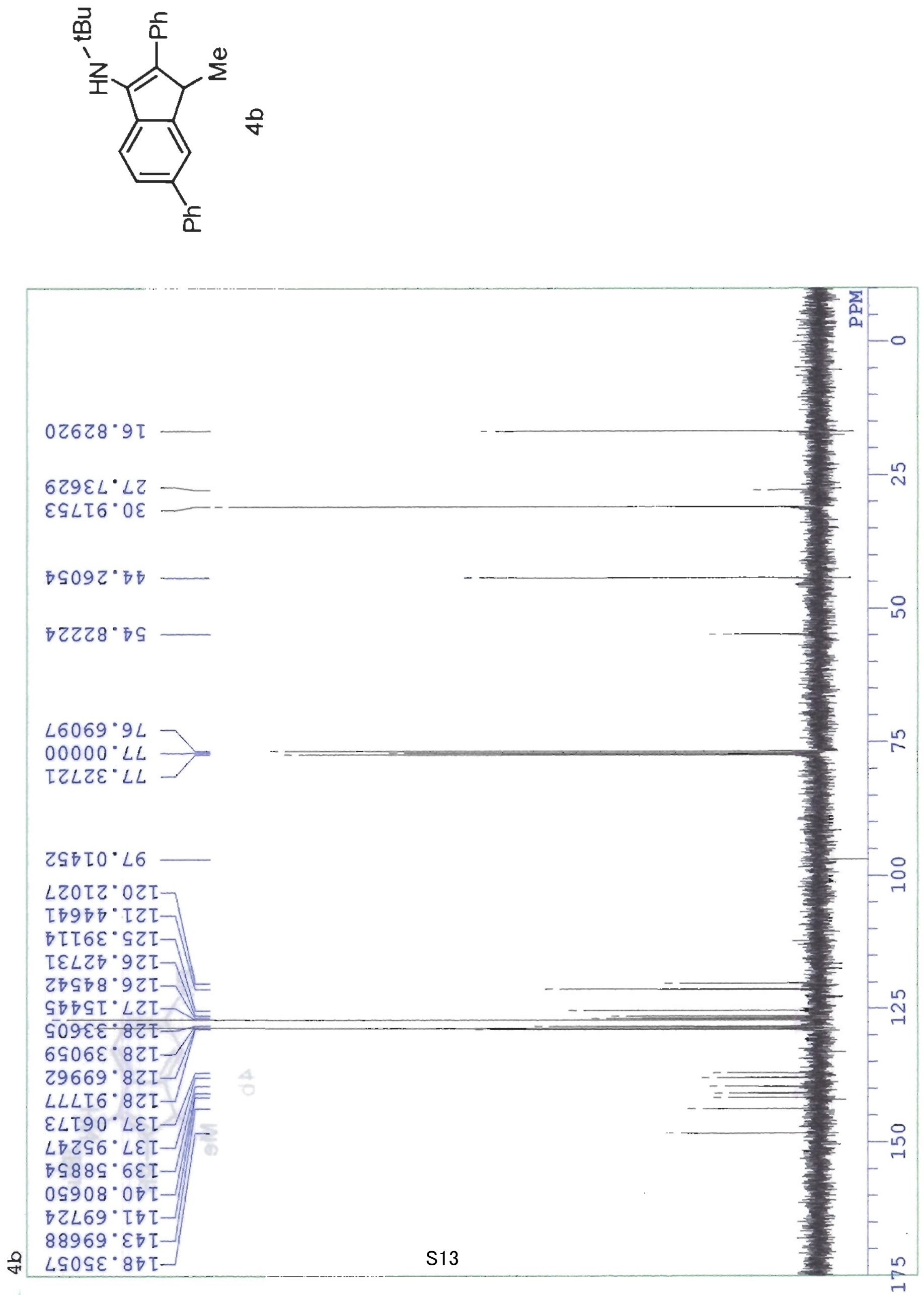

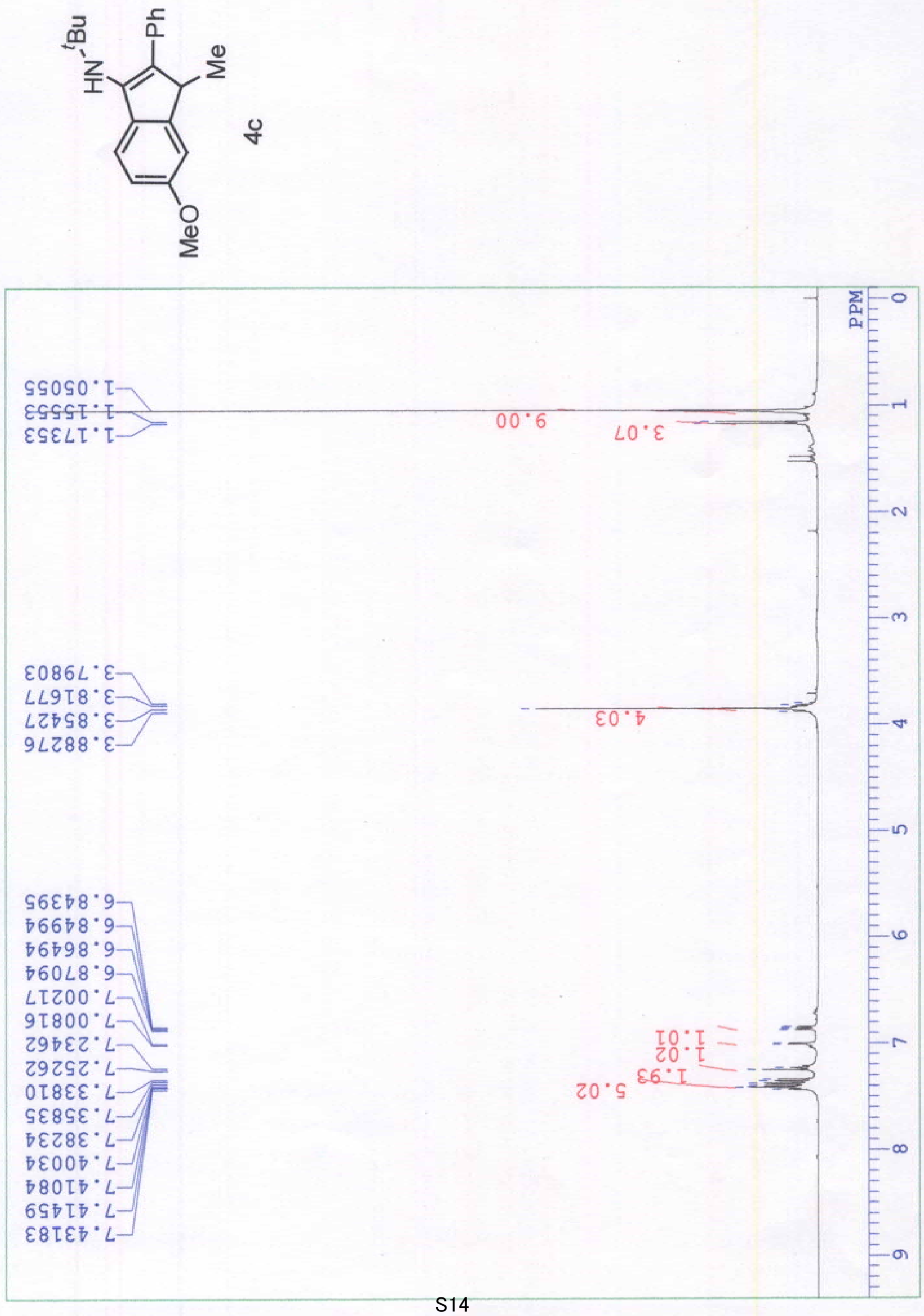

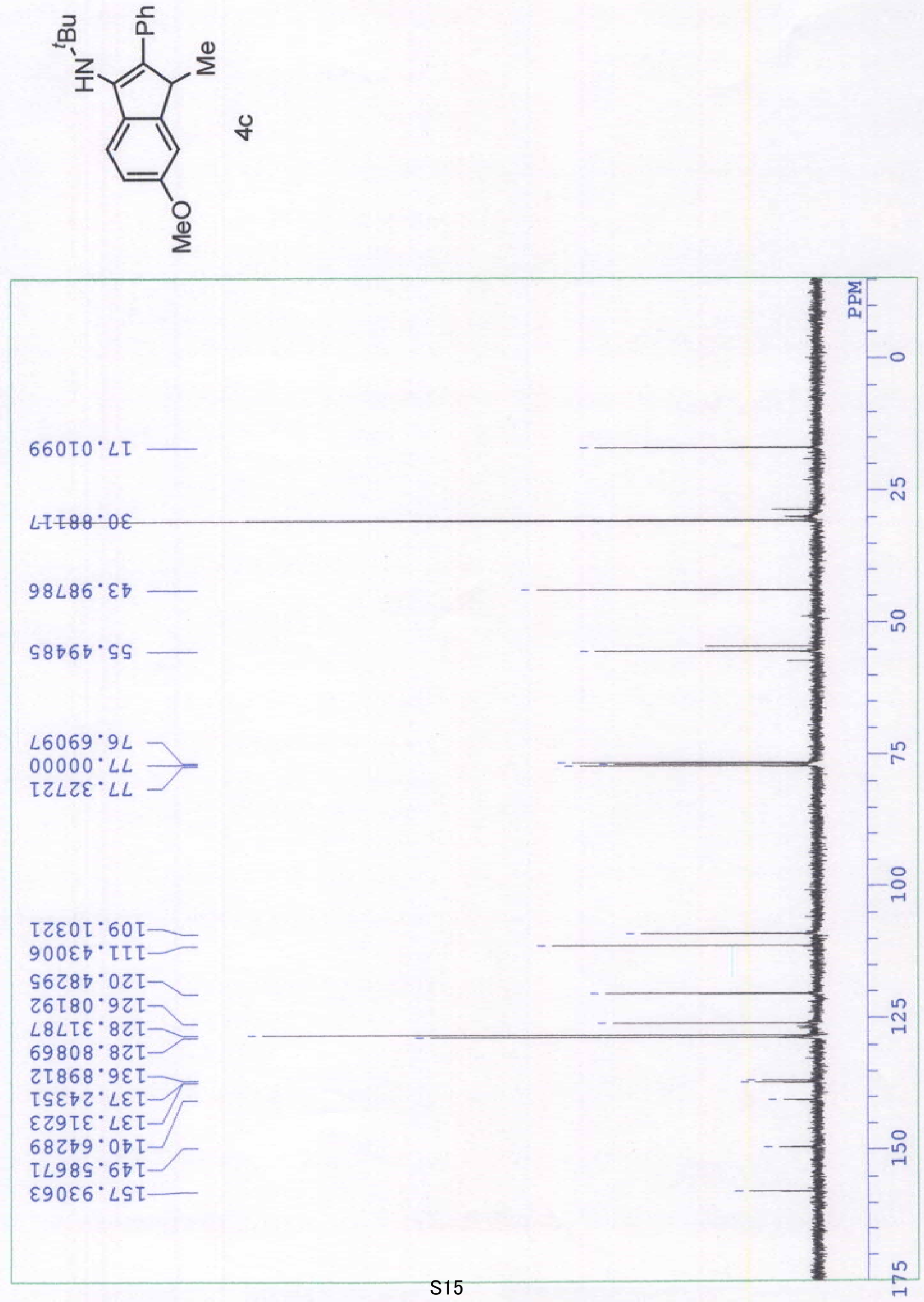

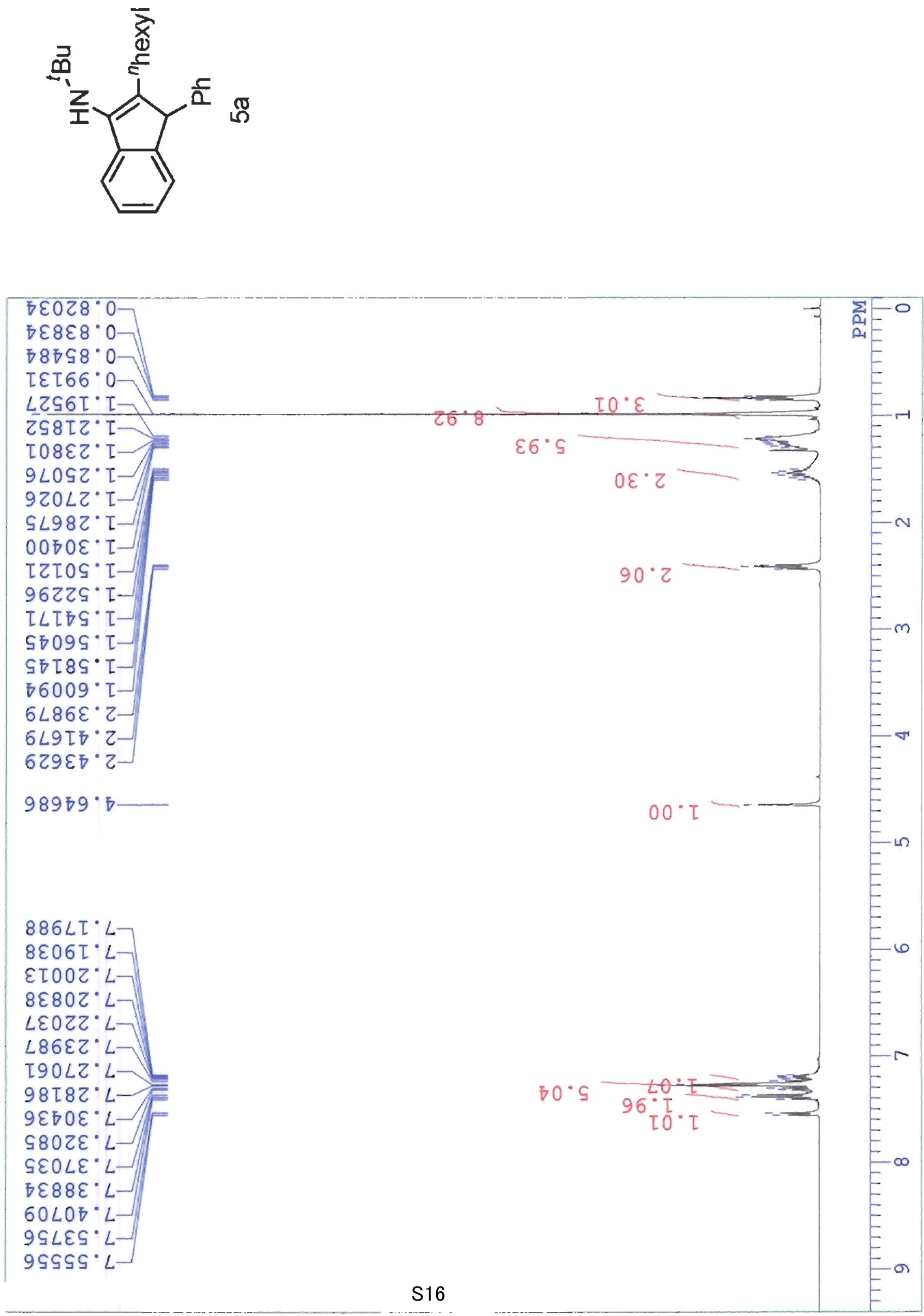

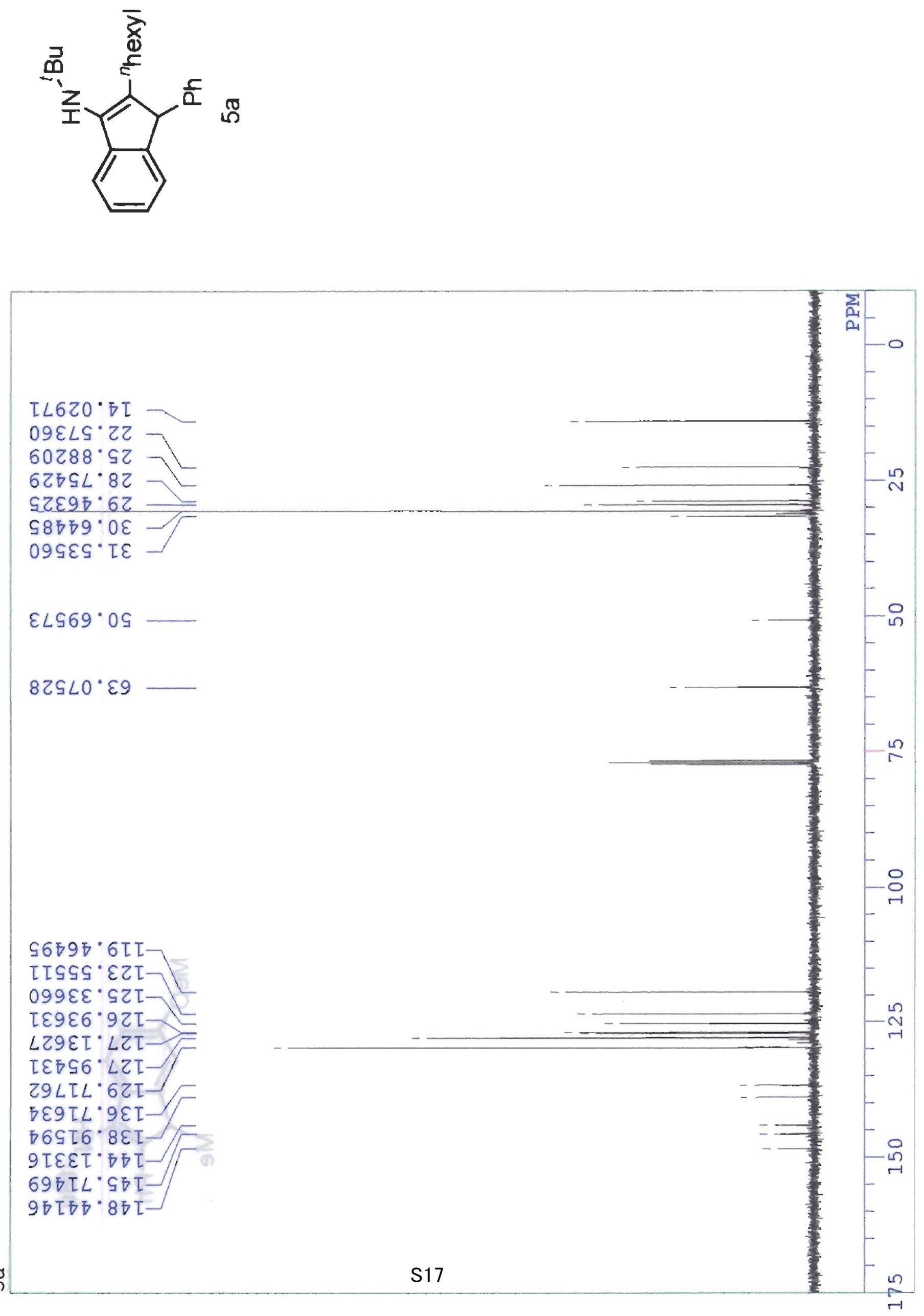

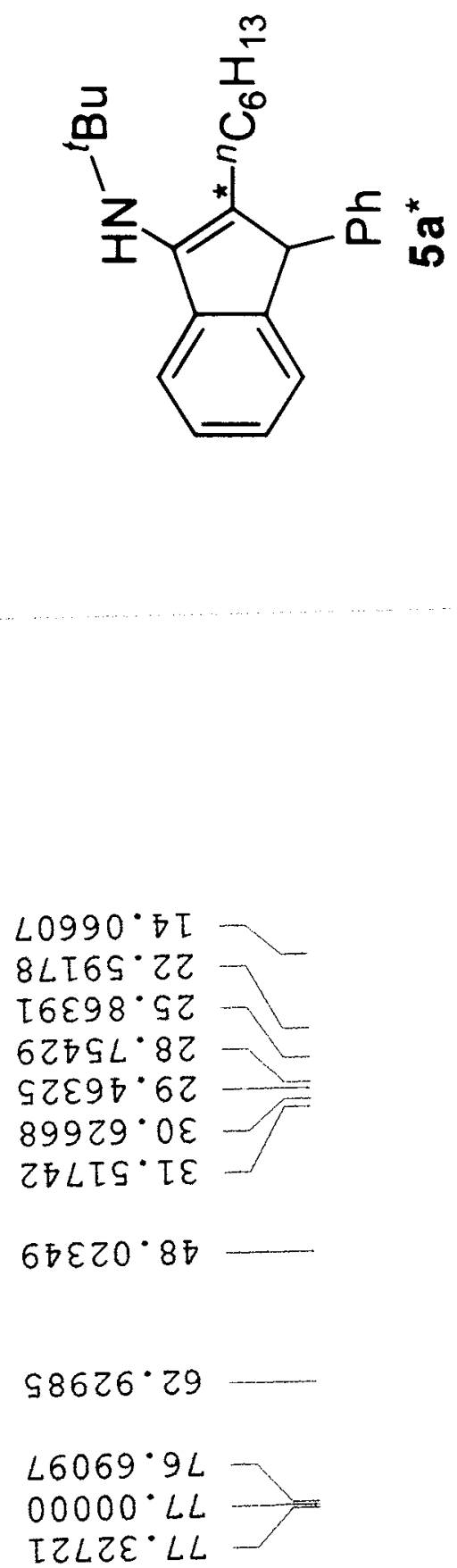

$96790^{\circ} 6 \mathrm{IL}$


$099 \varepsilon \varepsilon^{\circ} \varsigma \tau \tau$ IE9\&6.9ZT $608 I I^{\circ} \angle Z I$ OE๐S6 $6^{\circ} \mathrm{LZI-}$ 9ZI89.6ZI $\varepsilon 6 \varsigma \tau 6^{\circ} 8 \varepsilon \tau$ $298 \angle 0^{\circ} \nabla \sigma \mathrm{L}$

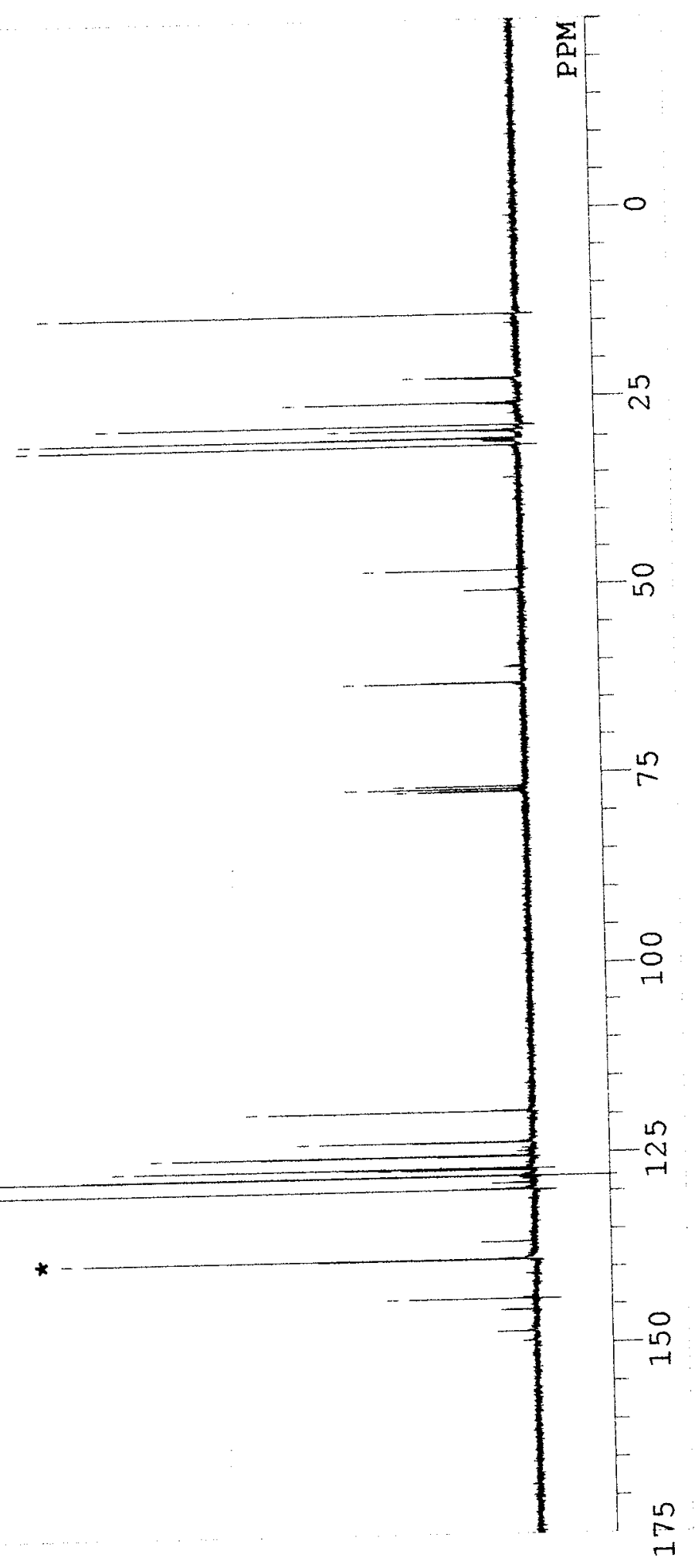



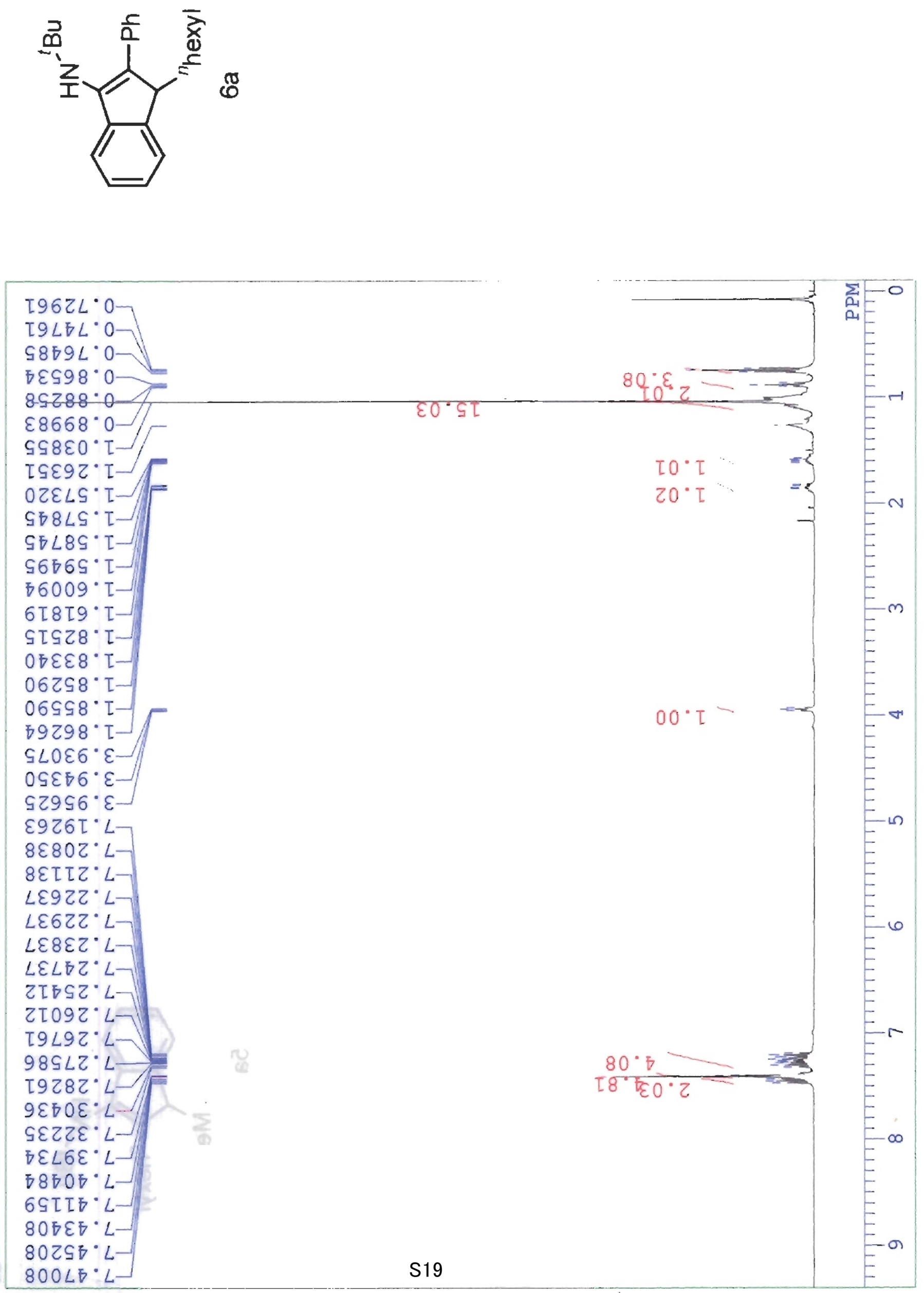

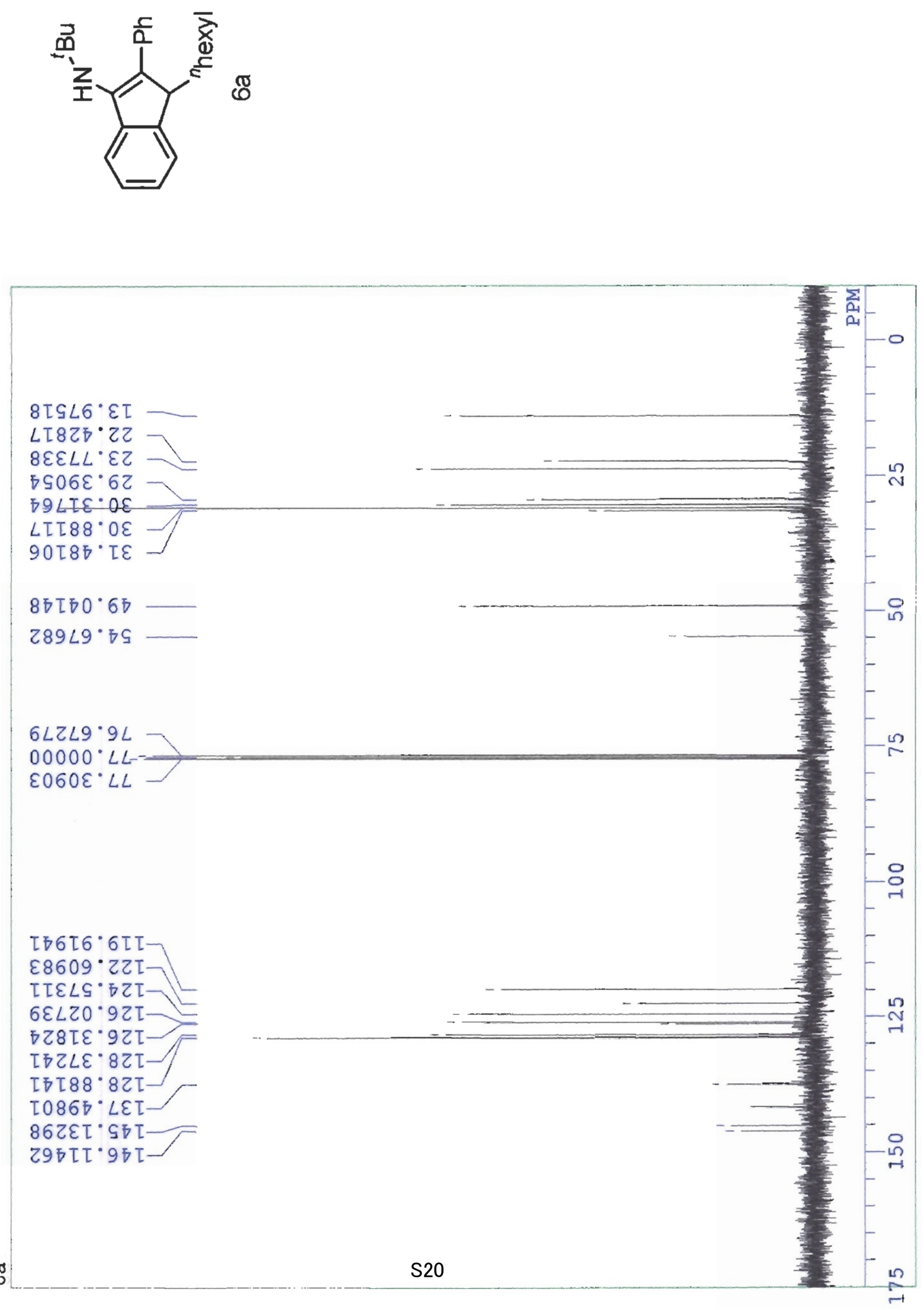

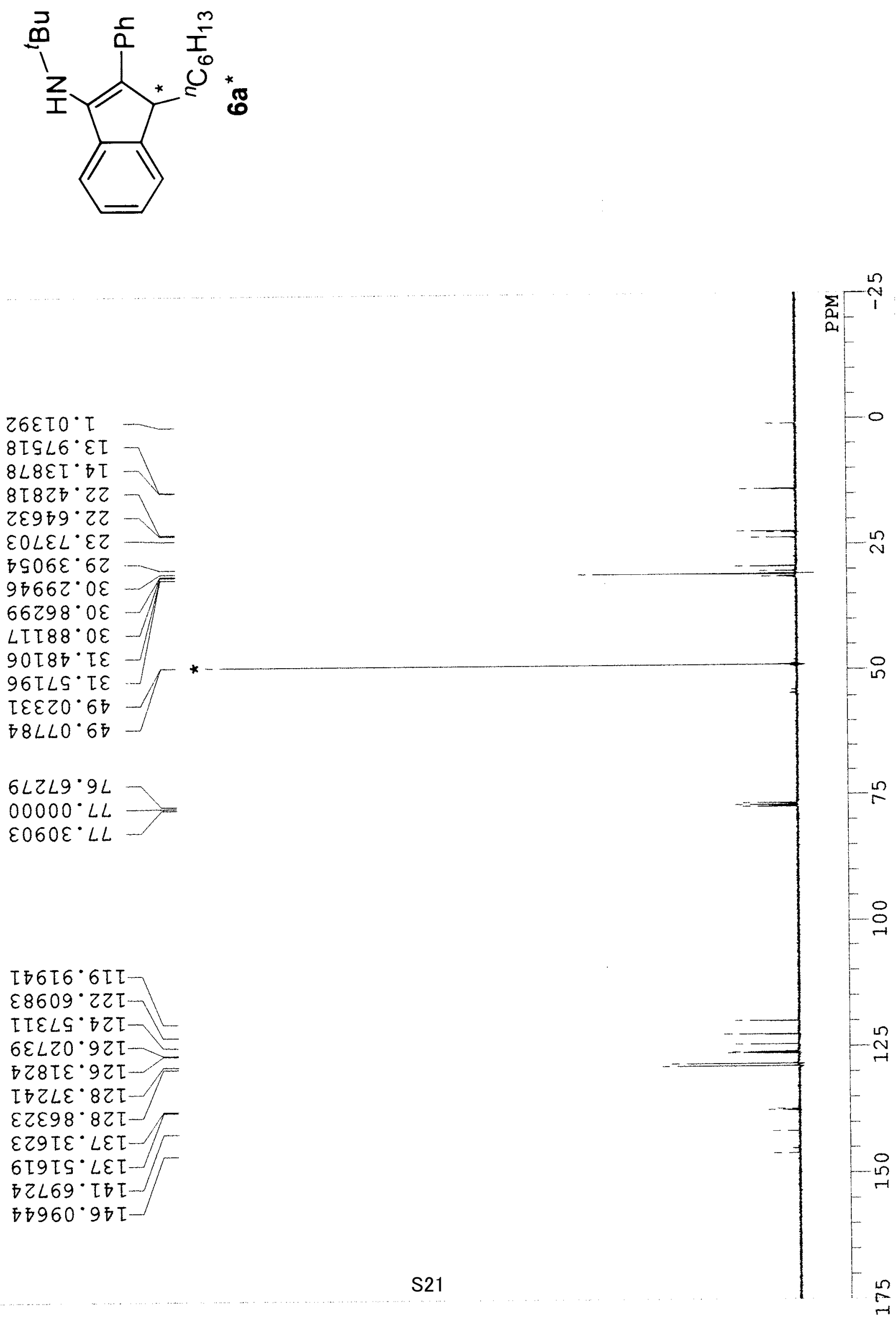